\title{
Holography of Wi-fi Radiation
}

\author{
Philipp M. Holl ${ }^{*}$ and Friedemann Reinhard \\ Technische Universität München, Walter Schottky Institut and Physik Department, \\ Am Coulombwall 4, 85748 Garching, Germany
}

(Received 25 September 2016; published 5 May 2017)

\begin{abstract}
Wireless data transmission systems such as wi-fi or Bluetooth emit coherent light—electromagnetic waves with a precisely known amplitude and phase. Propagating in space, this radiation forms a hologram - a two-dimensional wave front encoding a three-dimensional view of all objects traversed by the light beam. Here we demonstrate a scheme to record this hologram in a phase-coherent fashion across a meter-sized imaging region. We recover three-dimensional views of objects and emitters by feeding the resulting data into digital reconstruction algorithms. Employing a digital implementation of dark-field propagation to suppress multipath reflection, we significantly enhance the quality of the resulting images. We numerically simulate the hologram of a 10-m-sized building, finding that both localization of emitters and $3 \mathrm{D}$ tomography of absorptive objects could be feasible by this technique.
\end{abstract}

DOI: 10.1103/PhysRevLett.118.183901

Holography-three-dimensional imaging by phasecoherent recording of a two-dimensional wave front-is one of the most intriguing concepts of 20th century physics [1]. While most practical implementations have employed laser light, the concept itself is applicable to any kind of coherent radiation and was actually invented to improve electron microscopy. Other demonstrations have since been performed with sound waves [2], x rays [3], gamma rays [4], neutrons [5], and cold atoms [6].

It is an interesting question whether the omnipresent stray radiation of wireless devices forms holograms that encode three-dimensional views of the device and its surroundings. So far, holography of microwave radiation with similar (gigahertz) frequency has been demonstrated for the localization of radio-frequency emitters in a two-dimensional plane $[7,8]$ and near-field imaging with custom-built emitters $[9,10]$. However, efforts to obtain images from the stray radiation of unmodified wireless devices have remained limited to one-dimensional ranging [11].

Beyond its fundamental interest, indoor imaging by arbitrary wireless signals appears attractive for a variety of applications. These range from localization of radiofrequency tags in internet-of-things settings [7,8,12-14] over 3D motion capture for gaming [15-17] to throughwall imaging of moving targets for security enforcement [11,18-21]. Unfortunately, it is complicated by one major challenge: Multipath reflections-radiation scattered from walls and other surrounding objects outside the viewing area-blur radar signals and their echoes in indoor environments [14]. Existing approaches deal with this problem

Published by the American Physical Society under the terms of the Creative Commons Attribution 3.0 License. Further distribution of this work must maintain attribution to the author(s) and the published article's title, journal citation, and DOI. by producing or receiving short-pulse ultrawideband signals for time-domain ranging [22-24] or directed radiation for beam scanning and cancellation of multipath interference $[17,25,26]$. In doing so, they use only selected parts of the coherent radiation. To the best of our knowledge, imaging based on phase-coherent holographic recording of the full radiation field has remained elusive.

Here we demonstrate a holographic scheme to acquire three-dimensional images of building interiors from the radiation of an unmodified commercial narrow band wi-fi router. Our method does not require any prior knowledge of the emitted radiation and works with any type of signal, including encrypted communication.

Our approach is presented in Fig. 1. Additional details can be found in Supplemental Material [27]. We regard wi-fi radiation as coherent electromagnetic radiationelectromagnetic waves with well-defined amplitude $I$ and phase $\phi$. In this picture, a two-dimensional wave front $I(x, y) e^{i \phi(x, y)}$ at any plane in space represents a hologram [1]. It encodes a three-dimensional view of all objects traversed by the light beam, which can be recovered by digital reconstruction [28]. We record such holograms of wi-fi radiation by scanning an antenna across a meter-sized two-dimensional plane ("scanning antenna" in Fig. 1) pointwise registering received wi-fi signals $I(t)$ in the time domain by an oscilloscope [Rigol DS4034 + analog demodulation, $350 \mathrm{MHz}$ bandwidth; see Supplemental Material [27] for details and Fig. 2(a) for data].

We obtain the complex amplitude $\left.I e^{i \Phi}\right|_{x, y}$ by a homodyne scheme, which is presented in Figs. 2(a) and 2(b). In Fourier space, the propagation of the emitted complex signal $\widehat{I_{\mathrm{em}}(f)}$ to the point $x, y$ is described as $U(x, y, f) \widehat{I_{\mathrm{em}}(f)}$, where $U(x, y, f)$ denotes the propagation operator which includes amplitude attenuation and phase 


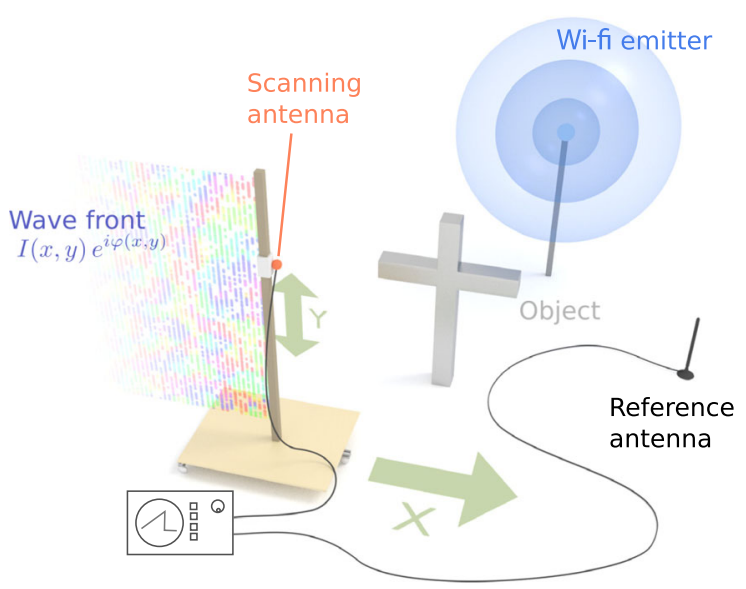

FIG. 1. Experimental setup. Stray radiation from a commercial router is employed to image meter-sized objects (gray cross) by digital holography. We record a hologram of wi-fi radiation by a synthetic aperture approach. A wi-fi antenna (scanning antenna) is moved across a 3 by 2 meter plane, pointwise registering wi-fi signals. The signal phase is recovered by a homodyne scheme, normalizing the signals to the signal of a stationary antenna (reference antenna).

delay. As the emitter is essentially a black box, $I_{\mathrm{em}} \widehat{(f)}$ is a priori unknown. To obtain the complex field, we therefore normalize the data using the signal received by a stationary reference antenna at position $x_{r}, y_{r}$ (Fig. 1), defining

$$
\begin{aligned}
\left.I(f) e^{i \Phi(f)}\right|_{x, y} & =\left.\widehat{I(f) \mid}\right|_{x, y} \stackrel{\text { daf }}{=} \frac{U(x, y, f) I_{\mathrm{em}}(f)}{U\left(x_{r}, y_{r}, f\right) I_{\mathrm{em}}(f)} \\
& =\frac{\mathcal{F}\left\{\left.I(t)\right|_{x, y}\right\}}{\mathcal{F}\left\{\left.I(t)\right|_{x_{r}, y_{r}}\right\}} .
\end{aligned}
$$

Here, $\mathcal{F}$ denotes the temporal Fourier transform.

This scheme recovers the amplitude $I$ and phase $\Phi$ of the propagated wave front up to an irrelevant constant factor $U\left(x_{r}, y_{r}, f\right)^{-1}$ for all frequencies $f$ within the wi-fi channel employed [Fig. 2(b)]. Repeating this analysis for every pixel $(x, y)$ yields a hologram as displayed in Figs. 2(c) and 2(d), a 2D map of both phase and amplitude for each frequency $f$. All experimental data sets in this work have been recorded in a closed room with other objects and metallic walls and are therefore strongly affected by standing waves from multipath reflection. We benchmark their strength from a single line of a 2D scan [Fig. 2(e)]. The amplitude modulation has a standing wave ratio of $\mathrm{SWR}=9.7$. It creates artifacts in the recovered phases, which correlate with peaks and valleys of the amplitude.

We now turn to the three-dimensional reconstruction of images from these holographic data. All following analysis is based on a data set, which has been recorded in the setting of Fig. 3(a). We capture a metallic (a) Wi-fi signals

(b) Signal correlation

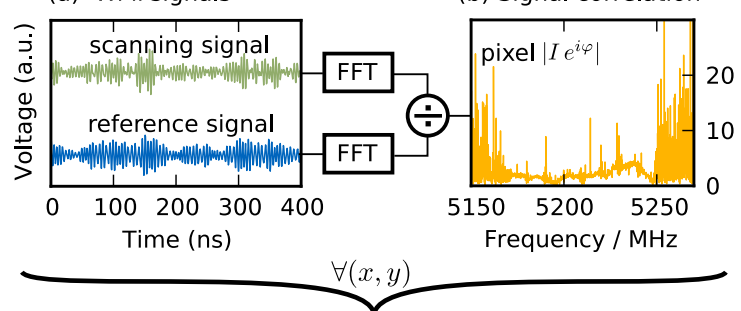

(c) Phase array

(d) Amplitude array
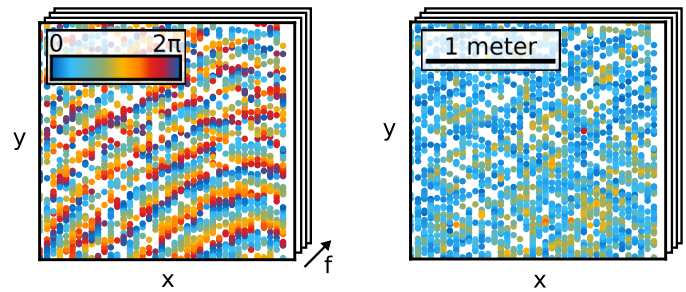

(e) Line scan

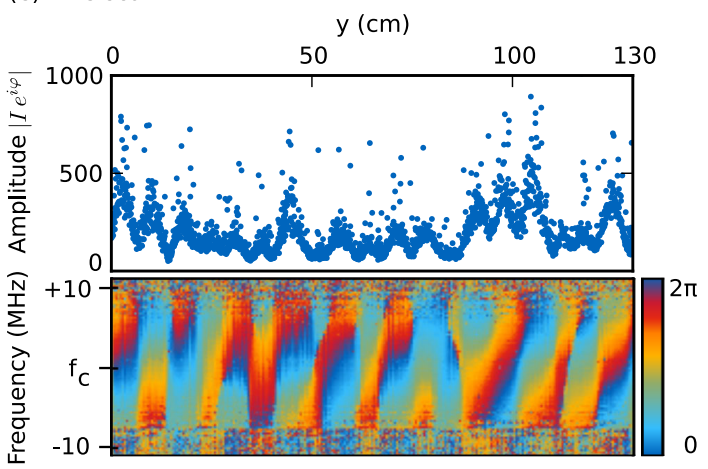

FIG. 2. Homodyne scheme for phase recovery. (a) The timedomain signals of the scanning and stationary antenna are Fourier transformed and (b) normalized to each other to obtain amplitude attenuation $I$ and the phase delay $\Phi$ imparted by the propagation for every frequency $f$ within the wi-fi bandwidth. (c),(d) Holograms are obtained by doing this for every pixel. (e) Multipath reflections in the building lead to a strong modulation with VSWR $=9.7$ and correlated artifacts in the phase. $f_{c} \approx 2.4 \mathrm{GHz}$ denotes the carrier frequency. (a)-(d) have been recorded using a $5 \mathrm{GHz}$ wi-fi emitter, and (e) using a $2.4 \mathrm{GHz}$ emitter.

cross-shaped phantom object. The illumination source is a commercial $5 \mathrm{GHz}$ wi-fi emitter (TP-LINK Archer C20, $802.11 \mathrm{ac}$ ), placed at a distance of $z_{e}=230 \mathrm{~cm}$ from the recorded plane, $90 \mathrm{~cm}$ behind the object plane $z_{o}$. Image reconstruction is performed by numerical backpropagation. This scheme recovers the light field at an arbitrary depth $z$ by propagating the recorded holographic wave front from $z_{0}$ in space according to the angular-spectrum relation [29,30]]

$$
\begin{aligned}
I(x, y, z)= & \mathcal{F}^{-1}\left[\exp \left(\mp i \frac{2 \pi\left(z-z_{0}\right)}{\lambda} \sqrt{1-\lambda^{2} f_{x}^{2}-\lambda^{2} f_{y}^{2}}\right)\right. \\
& \left.\times \mathcal{F}\left[I\left(x, y, z_{0}\right)\right]\right] .
\end{aligned}
$$


Here, $\mathcal{F}$ and $\mathcal{F}^{-1}$ denote the spatial Fourier transform of the light field and its inverse, respectively, and $f_{x}, f_{y}$ the transform's spatial frequencies. The sign in the exponential function is chosen negative by default and positive for $1-\lambda^{2} f_{x}^{2}-\lambda^{2} f_{y}^{2}<0$ to prevent an exponential blowup of evanescent waves. Backpropagation for a range of $z$ yields a 3D picture of all emitters and objects traversed by the emitted beams.

The most prominent feature in the recovered $3 \mathrm{D}$ view is the emitter [Figs. 3(b)-3(d)], which appears as a bright spot at the expected distance $\left(z_{e}=230 \mathrm{~cm}\right)$. Its extension $\left(\sigma_{x}=3 \mathrm{~cm}\right)$ is close to the diffraction limit set by the size of the recording plane and comparable to the wi-fi wavelength, allowing for centimeter-scale localization of its position in 3D space. Remarkably, this high accuracy appears unaffected by the presence of multipath reflections. We explain this effect by the fact that the freely propagating part of the radiation converges to a single point at $z_{e}$, while all scattered radiation loses its spatial information, resulting in an unspecific extended interference pattern of speckles at $z_{e}$. This view is supported by the observation that the emitter focus [bright spot in Figs. 3(b)-3(d)] contains only $15 \%$ of the received intensity.

We further reduce the intensity of speckles by incoherent white-light holography [31] [Fig. 3(d)], summing the backpropagated light fields of every frequency $f$ as $I(x, y)=\sum_{f}|I(x, y, f)|^{2}$. This procedure suppresses speckle interference by exploiting the fact that wi-fi radiation actually is white light. This is due to its modulation with bit-patterned data, which reduces the coherence length to a value set by the inverse signal bandwidth $\Delta f$, (a) Experimental setup

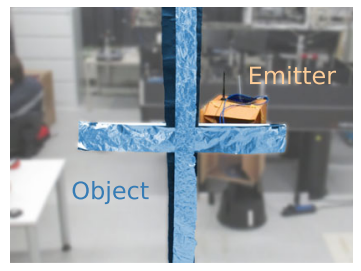

(c) Single frequency
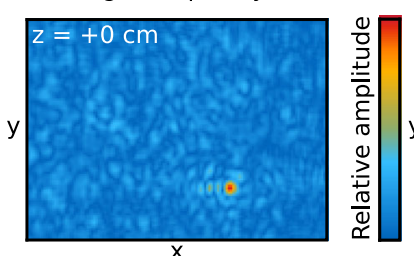

(d) White light

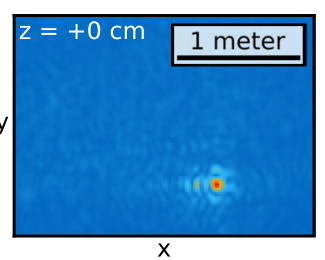

FIG. 3. Recovery of 3D holographic views by numerical backpropagation of the hologram. (a) A commercial router is used to illuminate a cross-shaped phantom object. (b) Backpropagation into the emitter plane reliably reveals the router as a single bright spot. (c) Multipath reflections create a speckle pattern that (d) can be canceled by incoherently averaging over holograms obtained at different transmission frequencies. in our case $(\Delta f \approx 70 \mathrm{MHz})$ to a numerical value of $\Delta x=c / \Delta f=4.3 \mathrm{~m}$. Our scheme can recover a whitelight image from reconstructions at each frequency, similar to filtering techniques in digital holography [32]. In contrast to some of these competing techniques [33], it does not degrade spatial resolution, since it preserves phase information for each frequency component upon acquisition and performs incoherent averaging only on the level of reconstructed images.

Objects in space are expected to appear as a shadow in the reconstruction of the emitted light cone. Indeed, a white-light reconstruction of the object plane $z_{O}$ reveals a shadow that correctly reproduces both the shape and dimensions of the cross-shaped phantom structure [Figs. 4(a) and 4(b)]. Speckles from multipath reflections are clearly visible despite the use of white-light suppression. Their detrimental effect on the image is stronger than in the case of the emitter plane (Fig. 3), since the signal intensity is now spread across a larger cross section.

We can further increase the imaging contrast by a digital implementation of dark-field propagation. This technique is commonly used in microscopy to reveal weakly absorbing objects. It reveals the scattered light field of an object by blocking out the illumination source by a suitable spatial filter.

Our digital version of the dark-field method eliminates the illumination source by masking the wi-fi source in the emitter plane, as shown in Fig. 4(a). The remaining field is then propagated to the object plane and subtracted from the directly reconstructed image to obtain

$\left|I_{\text {darkfield }}(x, y, z)\right|^{2}=\left|I_{\text {masked }}(x, y, z)\right|^{2}-\left|I_{\text {unmasked }}(x, y, z)\right|^{2}$.

By subtracting the two fields, one filters out the missing direct illumination which constitutes the dark field. Indeed, dark-field propagation reveals structures with a higher contrast, as can be seen from a comparison to direct reconstruction [Figs. 4(b) and 4(c)].

It is important to note that, with this method, points outside the dark-field cone are completely unphysical, which can be seen in Figs. 4(b)-4(d). As the emitter cannot be traced back to a single point but instead is spread out over a few centimeters, we use a Gaussian-shaped mask to block out its contribution. This comes at the disadvantage that a larger mask will produce a narrower dark-field beam. To overcome that problem, we block out only a part of the direct light. This results in a wider cone and produces a field that is approximately scaled by a constant.

Figure 4(d) shows reconstructions for different depths. The image of the structure appears blurred in out-of-focus planes, underlining the three-dimensional information contained in the hologram.

As a final outlook, we investigate the feasibility of a realistic large-scale reconstruction and tracking application 
(a) Dark-field propagation algorithm

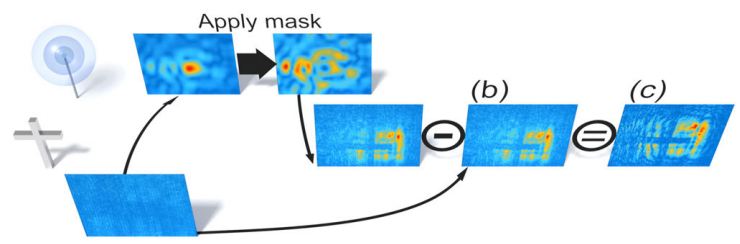

(b) Direct propgation

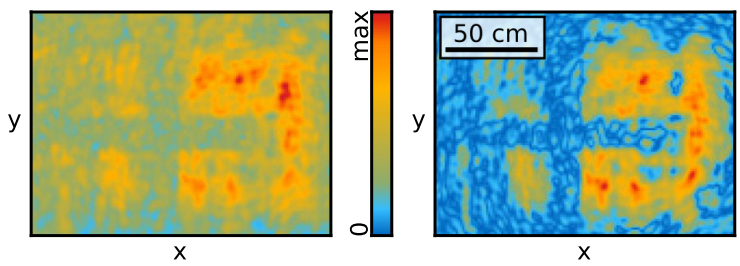

(d) Dark-field focus series

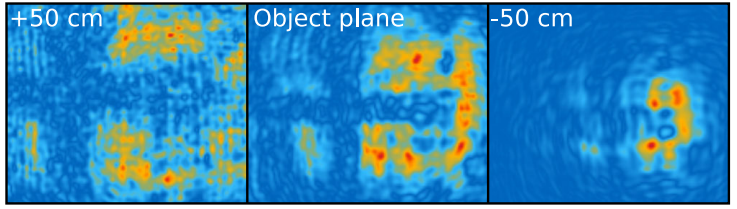

(e) Large-scale simulation
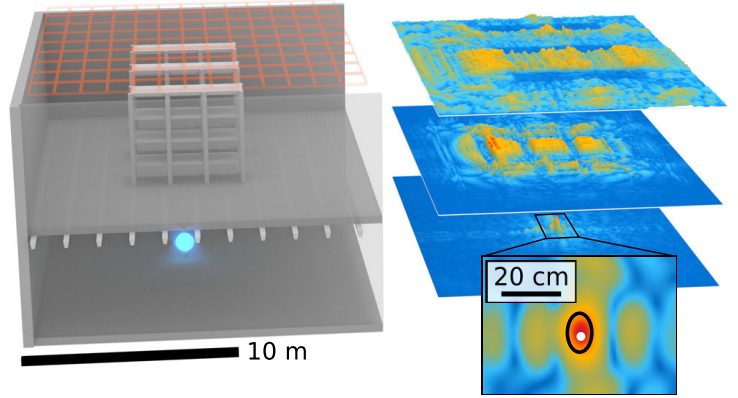

FIG. 4. Reconstruction of objects. (a) Sketch of the dark-field algorithm to enhance shadow contrast: A direct backpropagation into the object plane (b) is subtracted from the forward scattered radiation at this point, obtained by masking the emitter in its plane and forward propagating the result. (c) The resulting dark-field image; the contrast of objects is visibly enhanced. (d) Objects appear blurred in defocused planes, underlining the three-dimensional information contained in the hologram. (e) Simulation model of a full building on the left and reconstructed planes using our algorithm on the right. The black ring in the emitter plane shows the FWHM in intensity and the white dot the true emitter location.

[Fig. 4(e)]. Therefore, we performed a finite difference time domain simulation of the radiation field of a pointlike dipole emitter (frequency $f=2.4 \mathrm{GHz}$, bandwidth $\Delta f=20 \mathrm{MHz}$ ) on the floor of a $20 \times 17 \times 12 \mathrm{~m}^{3}$ storage building. We record a hologram across a twodimensional plane in the ceiling (orange grid), as it could be implemented in practice by a two-dimensional array of stationary antennas. As a model for objects, we add an intermediate floor at a height of $6 \mathrm{~m}$, supported by perfectly reflective metal bars, which supports a set of perfectly reflective metallic shelves. The three-dimensional reconstruction of the hologram clearly shows sharp outlines of the metallic shelves and bars in their respective reconstructed planes. The emitter location can be inferred with an accuracy of only $3 \mathrm{~cm}$, determined by the FWHM of intensity in its pointlike image, despite the presence of multipath reflections from the surrounding structures [27].

Several future improvements of the technique appear attractive. First, a stationary two-dimensional array of antennas could replace our slow synthetic aperture approach of mapping a wave front by pointwise scanning. Such a device could provide pictures several orders of magnitude faster. Specifically, video rate 3D imaging appears feasible, since a $1 \mathrm{GS} / \mathrm{s}$ acquisition device could acquire a 100-ns-scale snapshot of signals for one million pixels at a rate of 10 frames per second. Second, future wireless signals will significantly improve image quality. Their higher bandwidth will reduce speckle contamination, while their higher frequencies will increase spatial resolution, to the $5 \mathrm{~mm}$ scale for $60 \mathrm{GHz}$ transmission currently under discussion (IEEE 802.11ad).

The implications of this result are manifold. It opens a path to three-dimensional imaging and localization in the presence of multipath reflections, which does not require ultrawideband signals or directed emitters. Its power in 3D localization of sources could find applications in indoor navigation, where it could track radio-frequency labels even in cluttered indoor settings, potentially at video rate acquisition speeds. It equally raises concerns about the privacy of wireless communication: Even encrypted communication transmits a three-dimensional picture of its surrounding to the outer world, which can be recovered by suitable strategies.

This work has been supported by the Deutsche Forschungsgemeinschaft (Emmy Noether Grant No. RE 3606/1-1) and by the Technische Universität München.

*Corresponding author.

Philipp.Holl@wsi.tum.de

†Friedemann.Reinhard@wsi.tum.de

[1] D. Gabor, A new microscopic principle, Nature (London) 161, 777 (1948).

[2] R. K. Mueller and N. K. Sheridon, Sound holograms and optical reconstruction, Appl. Phys. Lett. 9, 328 (1966).

[3] M. Tegze and G. Faigel, X-ray holography with atomic resolution, Nature (London) 380, 49 (1996).

[4] P. Korecki, J. Korecki, and T. Ślęzak, Atomic Resolution Gamma-Ray Holography Using the Mössbauer Effect, Phys. Rev. Lett. 79, 3518 (1997).

[5] B. Sur, R. B. Rogge, R. P. Hammond, V. N. P. Anghel, and J. Katsaras, Atomic structure holography using thermal neutrons, Nature (London) 414, 525 (2001).

[6] M. Morinaga, M. Yasuda, T. Kishimoto, F. Shimizu, J. I. Fujita, and S. Matsui, Holographic Manipulation of a Cold Atomic Beam, Phys. Rev. Lett. 77, 802 (1996). 
[7] R. Miesen, F. Kirsch, and M. Vossiek, in Proceedings of the 2011 IEEE International Conference on RFID (IEEE, New York, 2011), p. 32, DOI: 10.1109/RFID.2011.5764633.

[8] R. Miesen, F. Kirsch, and M. Vossiek, UHF RFID localization based on synthetic apertures, IEEE Trans. Autom. Sci. Eng. 10, 807 (2013).

[9] R. K. Amineh, M. Ravan, A. Khalatpour, and N. K. Nikolova, Three-dimensional near-field microwave holography using reflected and transmitted signals, IEEE Trans. Antennas Propag. 59, 4777 (2011).

[10] A. Popov, I. Prokopovich, and D. Edemskii, in Proceedings of 2016 Days of Diffraction (IEEE, New York, 2016), pp. 340-345, DOI: 10.1109/DD.2016.7756870.

[11] K. Chetty, G. E. Smith, and K. Woodbridge, Through-thewall sensing of personnel using passive bistatic wifi radar at standoff distances, IEEE Trans. Geosci. Remote Sens. 50, 1218 (2012).

[12] C. Hekimian-Williams, B. Grant, X. Liu, Z. Zhang, and P. Kumar, in Proceedings of the 2010 IEEE International Conference on RFID (IEEE, New York, 2010), p. 89.

[13] J. Zhou and J. Shi, RFID localization algorithms and applications-A review, Journal of Intelligent Manufacturing 20, 695 (2009).

[14] J. Wang and D. Dude Katabi, Dude, where's my card?: RFID positioning that works with multipath and non-line of sight, Comput. Comm. Rev. 43, 51 (2013).

[15] J. Wang, D. Vasisht, and D. Katabi, RF-IDraw: Virtual touch screen in the air using RF signals, Comput. Comm. Rev. 44, 235 (2014).

[16] H. Abdelnasser, M. Youssef, and K. A. Harras, in Proceedings of the 2015 IEEE Conference on Computer Communications INFOCOM (IEEE, New York, 2015), pp. 1472-1480, DOI: 10.1109/INFOCOM.2015.7218525.

[17] F. Adib, C.-Y. Hsu, H. Mao, D. Katabi, and F. Durand, Capturing the human figure through a wall, ACM Trans. Graph. 34, 219 (2015).

[18] E. J. Baranoski, Through-wall imaging: Historical perspective and future directions, J. Franklin Inst. 345, 556 (2008).

[19] M. Bocca, O. Kaltiokallio, N. Patwari, and S. Venkatasubramanian, Multiple target tracking with rf sensor networks, IEEE Trans. Mob. Comput. 13, 1787 (2014).

[20] S. Nannuru, Y. Li, Y. Zeng, M. Coates, and B. Yang, Radiofrequency tomography for passive indoor multitarget tracking, IEEE Trans. Mob. Comput. 12, 2322 (2013).
[21] J. Wilson and N. Patwari, See-through walls: Motion tracking using variance-based radio tomography networks, IEEE Trans. Mob. Comput. 10, 612 (2011).

[22] Y. Yang and A. E. Fathy, in Proceedings of the 2005 IEEE Antennas and Propagation Society International Symposium (2005), Vol. 3B, p. 334, DOI: 10.1109/APS.2005.1552508.

[23] C. Le, T. Dogaru, L. Nguyen, and M. A. Ressler, Ultrawideband (UWB) radar imaging of building interior: Measurements and predictions, IEEE Trans. Geosci. Remote Sens. 47, 1409 (2009).

[24] A. Beeri and R. Daisy, High-resolution through-wall imaging. Proc. SPIE Int. Soc. Opt. Eng. 6201, 62010J (2006).

[25] F. Adib and D. Katabi, in Proceedings of ACM SIGCOMM 2013 Conference (Association for Computing Machinery, New York, 2013), p. 75, DOI: 10.1145/2486001.2486039.

[26] D. Huang, R. Nandakumar, and S. Gollakota, in Proceedings of the 12th ACM Conference on Embedded Networked Sensor Systems (Association for Computing Machinery, New York, 2014), pp. 266-279, DOI: 10.1145/2668332.2668344.

[27] See Supplemental Material at http://link.aps.org/ supplemental/10.1103/PhysRevLett.118.183901 for details on data acquisition, analysis and simulation.

[28] U. Schnars and W.P. O. Jüptner, Digital recording and numerical reconstruction of holograms, Meas. Sci. Technol. 13, R85 (2002).

[29] G. Pedrini, W. Osten, and Y. Zhang, Wave-front reconstruction from a sequence of interferograms recorded at different planes, Opt. Lett. 30, 833 (2005).

[30] L. Mandel and E. Wolf, Optical Coherence and Quantum Optic. (Cambridge University Press, Cambridge, England, 1995).

[31] D. N. Naik, G. Pedrini, and W. Osten, Recording of incoherent-object hologram as complex spatial coherence function using Sagnac radial shearing interferometer and a Pockels cell, Opt. Express 21, 3990 (2013).

[32] D. N. Naik, G. Pedrini, M. Takeda, and W. Osten, Spectrally resolved incoherent holography: 3D spatial and spectral imaging using a Mach-Zehnder radial-shearing interferometer, Opt. Lett. 39, 1857 (2014).

[33] D. T. Dul and P. Korecki, Wavelet analysis of white beam X-ray fluorescence holograms: Determination of lattice sites and imaging of local atomic structure, New J. Phys. 14, 113044 (2012). 